\title{
Evaluation of satellite-based precipitation over Tianshan mountainous
}

\author{
Chuancheng Zhao \\ Institute of Information and Engineering, Lanzhou City \\ University \\ Lanzhou Gansu, China \\ State Key Laboratory of Cryospheric Science, CAREERI, \\ CAS \\ Lanzhou Gansu, China \\ Cold and Arid Regions Environmental and Engineering \\ Research Institute, Chinese Academy of Sciences \\ Lanzhou Gansu, China \\ Zhao_chch1978@163.com
}

\section{Shuxia Yao}

Institute of mathematic, Lanzhou city university

\author{
Lanzhou Gansu, China
}

yaoshuxia@163.com

Jian Wang

Cold and Arid Regions Environmental and Engineering Research Institute, Chinese Academy of Sciences

\section{Lanzhou Gansu, China}

wjshuigong@lzb.ac.cn

Jianbo Cui

Institute of Information and Engineering, Lanzhou City University

Lanzhou Gansu, China

gscjb@vip.163.com

\begin{abstract}
The availability of in situ measurements of precipitation in remote locations, especially in mountainous regions of northwest of China, is limited. The ground-based hydrometeorological observations are frequently sparse in remote mountainous regions and the situation is not improving. While hydrological models have the potential to provide the necessary information, without adequately accurate climate (rainfall, evaporation, etc.) input information, it is extremely difficult to establish models and generate representative water resource availability information. As a result, the use of satellite measurements of precipitation is attractive for water resources management. However, these products have several weaknesses, for example their failure to capture certain types of precipitation, limited accuracy and limited spatial and temporal resolution. This paper evaluates the usefulness of TRMM 3B43 precipitation products over data scarce of complex mountainous terrain in northwest China from a water resources perspective. It is clear that the satellite data cannot be used directly in conjunction with historical gauge data. Specifically, the satellite data do not reflect the strong influences on precipitation of topography in some of the basins. However, the prospects of applying relatively straightforward adjustments are promising and further assessments appear to be justified.
\end{abstract}

Index Terms-Precipitation, TRMM Satellite, Tianshan mountainous, Spatial Distribution.

\section{INTRODUCTION}

Accurate measurements of precipitation on a variety of space and time scales are important not only to weather forecasters and climate scientists, but also to a wide range of decision makers, including hydrologists, agriculturalists, emergency managers, and industrialists[1]. Mountainous regions are generally data-sparse, yet display extremely complex weather pattens and climatologies. Mountains exert strong influence on airflow patterns, storm rainfall intensity, and cause great spatial variability [2]. In mountain regions, in addition to the stochastic nature of rainfall, the precipitation pattern may be influenced by the irregular topography. The large variability in altitude, slope and aspect may increase variability by means of processes such as rain shading and strong winds. The best method to improve the quality of spatial rainfall estimation is to increase the density of the monitoring network. However, this is very costly, and in many cases practically infeasible. And even for dense net-works, interpolation remains necessary in order to calculate the total rainfall over a certain area [3].

Precipitation, the primary input to hydrological models, shows a considerable spatial variation. This is brought about by differences in the type and scale of development of precipitation producing processes, and strongly influenced by local or regional factors, such as topography and wind direction at the time of precipitation. The problem is to try to describe the spatial variation and to make estimates of precipitation in areas where there are no monitoring stations. Resources for collecting such basic information are limited particularly in the remote mountainous regions. 
Measured data from rain gauge networks are still conventionally the most reliable source of area-averaged precipitation for the land surface of the earth. However, rain gauge measurement networks are not as dense or regular as in mountainous regions. Interpolation from ground-based point data is main approaches to estimate precipitation. While these data do not direct incorporate the additional information by the gridded. In recent years, the development of weather radar systems and the advancements in remote sensing techniques have provided rainfall data at higher spatial resolutions. The evaluation of satellite rainfall estimates was one application of the East Asian daily gauge analysis described by Xie et al. [4].

This paper describes a comprehensive evaluation of satellite-based precipitation data with rain gauge based of monthly precipitation over Tianshan Mountainous, located in the northwest of China where is a typical arid and semi-arid region, stretch from west to east, divide Xinjiang into two parts from 1998 to 2010. Intercomparisons and cross-validation tests have been carried out between TRMM satellite precipitation data and independent rain gauge.

\section{MATERIALS AND METHODS}

\section{A. Study area}

Tianshan Mountainous, located in the northwest of China where is a typical arid and semi-arid region, stretch from west to east, divide Xinjiang into two parts, i.e. Southern Xinjiang and Northern Xinjiang[5]. Situated in the hinterland of Eurasian continent, and far away from the ocean, Precipitation is the main source of surface water to supply for river runoff, farm irrigation and drinking. In southern of Tianshan mountainous, the southwest monsoon from the Indian Ocean is difficult for vapour to reach mountainous regions due to intercepting by the Himalaya Mountains and the TsinghaiTibet Plateau. The southeast monsoon which mainly impacts eastern China, rarely reaches mountainous regions. Tianshan mountainous lie in the prevailing westerlies with the west wind current as the major vapour resource. The dry, cold current of the Arctic Ocean is the second resource, and its vapour content is equal to $25-33 \%$ of that of the west wind current. The pattern of Tianshan mountainous present more precipitation in the north than in the south, more in the west than in the east, more in the mountain area than in the plain area and more on the windward slope than on the leeward slope. Zonal vertical distribution of mountainous precipitation is also obvious [6].

\section{B. Data Collection}

The TRMM satellite was launched on 27 November 1997 from Japan's Tanegashima launch facility. Fundamentally, the TRMM research programme is dedicated to measuring tropical-subtropical rainfall over a lengthy time period, and by doing so, acquiring the first accurate, representative and consistent ocean climatology of precipitation. TRMM was initially launched into a low-altitude $(350 \mathrm{~km})$, non-sun synchronous orbit inclined at 50 degrees to the Earth's equatorial plane, with a nominal mission lifetime of three years, but with expectations for a longer lifetime. Thus it has better ability to measure rainfall than other passive satellite remote sensing.

TRMM 3B43 is a monthly satellite rainfall data product of the NASA Goddard Space Flight Centre with a spatial resolution of $0.25^{\circ} \times 0.25^{\circ}$ (Huffman et al., 2007). The approach used in building the product is to use all available PMW estimates and fill any cells without a PMW estimate for a given time step using IR estimates that have been calibrated using the PMW and space-born precipitation radar data. The product is further calibrated against monthly gauge data to scale the estimates. The TRMM algorithm ensures that every grid box has the best possible estimate, but it also can result in a statistically heterogeneous dataset. TRMM 3B43 data used for the current study covers the period from January 1998 to December 2009. The dataset has shown good performance in different regions around the world. TRMM 3B43 data has two version, one is version 6 released since 1998, another is version 7 released in 2012.

Monthly values of precipitation between 1998 and 2010 is used for 32 stations located in the Tianshan mountainous considered. Many stations are distributed in low elevations from $35 \mathrm{~m}$ to $3539 \mathrm{~m}$ presented in Fig. 1, nonexistent stations above $4000 \mathrm{~m}$ over high mountainous. This data is provided by the National Climatic Centre of China, the China Meteorological Administration. The quality of the data was firmly controlled before its release and homogeneity tests were also performed. Although the number of stations with sufficiently long, quality-controlled, records is smaller than desired, they are still capable of representing the precipitation that falls in the Tianshan mountainous. The series of datasets are different in this research, comparisons are made over a 13year period between 1998 to 2010.

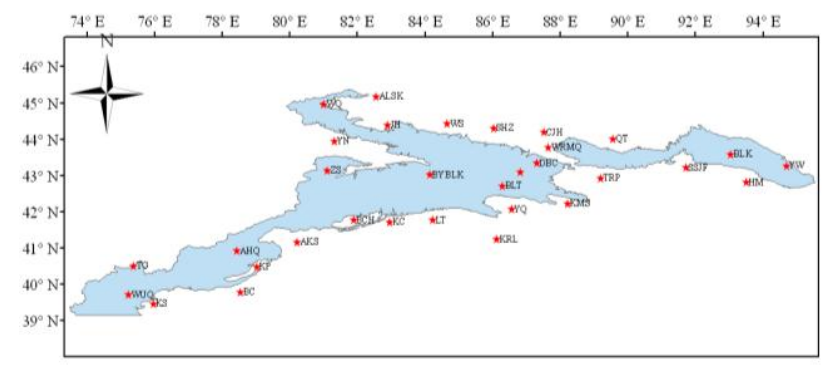

Fig. 1. Location of the study area and distribution of meteorological stations.

\section{Methods}

This research explores the use of TRMM precipitation data to detect changes in precipitation and investigates whether satellite precipitation estimates can adequately capture spatial and temporal variability when compared to rain gauge across Tianshan mountainous regions. Satellite-derived precipitation only estimate precipitation and are not direct measures of quantity. It is therefore important to establish the accuracy of satellite precipitation through validating estimates against ground measurements from rain gauges or radar observations.

The root-mean square error (RMSE) and and correlation coefficient $(\mathrm{R})$ are used to quantify the error in the TRMM 
3B43 precipitation estimates relative to the observation precipitation data. RMSE was used to measure the quantitative agreement between TRMM 3B43 and rain gauge data, which was calculated as:

$$
R M S E=\sqrt{\frac{1}{n} \sum_{i=1}^{n}\left(x_{i}-y_{i}\right)^{2}}
$$

where $n$ is the number of observations in the time series, $x_{i}$ is the rain gauge data and $\mathrm{y}_{i}$ is the TRMM 3B43 precipitation estimate for grid cell $i$.

\section{RESULTS AND DISCUSSION}

To evaluate the TRMM 3B43 version 6 (3B43_v6) data, correlation coefficients was firs calculated on annual between rain gauge and 3B43_v6 for each station and corresponding grid over Tianshan mountainous regions(Fig. 2a). The results indicated that the association between rain gauge and 3B43_v6 were statistically significant over whole study regions. Correlations were greatest in the mid with a maximum correlation coefficient of 0.95 , and lowest in the east and west at 0.45 . Then, RMSE is calculated based on formula1. Annual comparison of rain gauge and 3B43_v6 indicated large RMSE in the mid of study regions. Error values were greater in north than south, east than west (Fig. 2b). This is likely resultant of north receiving the more quantities of precipitation, so despite a high correlation coefficient a large RMSE also ensued. RMSF values indicated 3B43_v6 underestimate precipitation across much of study regions, with greatest accuracy predictions $(\mathrm{RMSF} \approx 1)$ in south which lower precipitation.

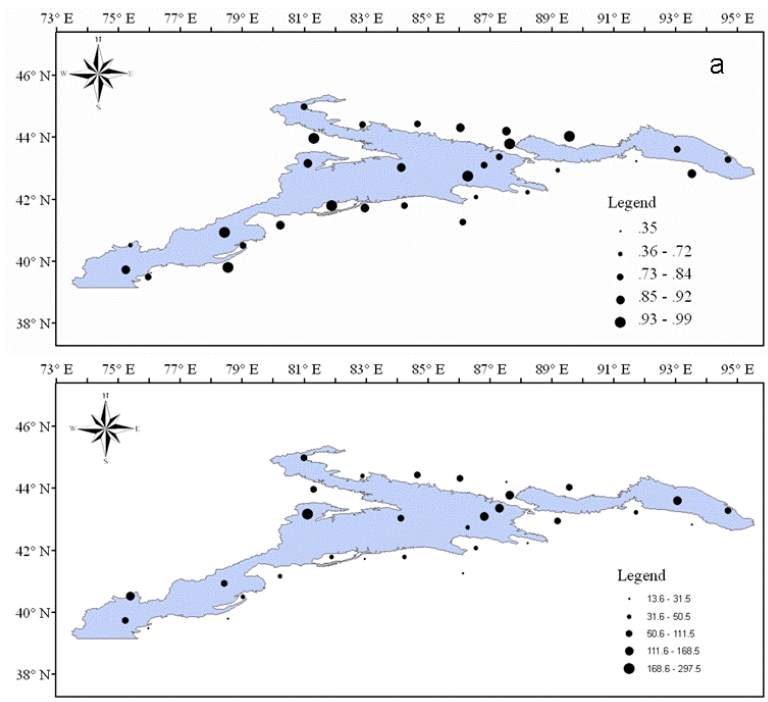

Fig. 2. Statistical comparison of rain gauge and 3B43 v6 annual time series between 1998 and 2010 (a) correlation coefficient, (b) RMSE.

To evaluate the TRMM 3B43 version 7 (3B43_v7) data, correlation coefficients was firs calculated on annual between rain gauge and 3B43_v7 for each station and corresponding grid over Tianshan mountainous regions(Fig. 3a). The results indicated that the association between rain gauge and 3B43_v7 were statistically significant over whole study regions.
Correlations were greatest in the mid with a maximum correlation coefficient of 0.98 , and lowest in the east and west at 0.72 . Then, RMSE is calculated based on formula1. Annual comparison of rain gauge and 3B43_v7 indicated large RMSE in the mid of study regions. Error values were greater in north than south (Fig. 3b). This is likely resultant of north receiving the more quantities of precipitation, so despite a high correlation coefficient a large RMSE also ensued. RMSF values indicated 3B43_v7 underestimate precipitation across much of study regions, with greatest accuracy predictions $(\mathrm{RMSF} \approx 1)$ in south which lower precipitation.

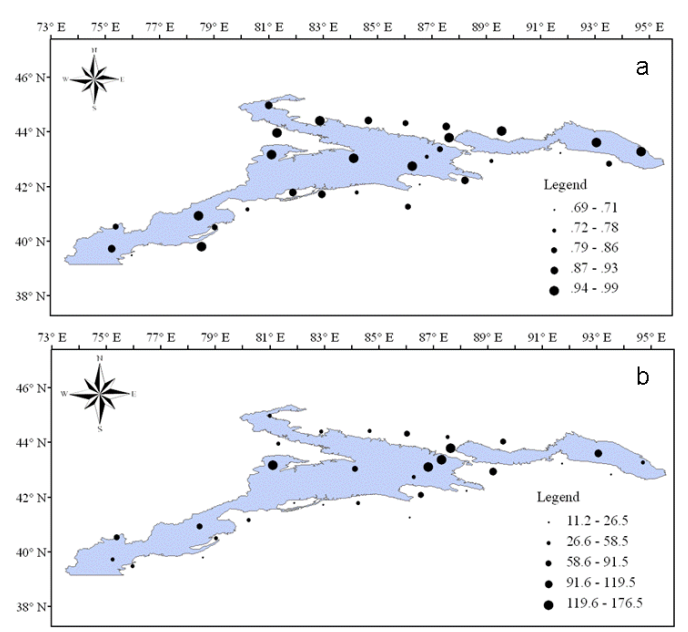

Fig. 3. Statistical comparison of rain gauge and 3B43 v7 annual time series between 1998 and 2010 (a) correlation coefficient, (b) RMSE.

Spatial distribution of statistics such as correlations and RMSE of precipitation values, provided a more complete picture of the precipitation estimations as well as of the observation performance in reproducing the satellite-based precipitation field. Although TRMM 3B43 clearly underestimate observed precipitation, they present good anomalies correlation results with observational data.

However, TRMM 3B43 data present lower precipitation values than the observational data. In general, higher errors in terms of discrepancies are obtained over the highest precipitation areas, namely northwards. The correlation analysis performed on precipitation anomalies has provided some measure of the similarity existing between evaluated and observational anomalies series. It shows time correlation values higher than 0.95 that indicate a good degree of resemblance between evaluated and observational data sets in terms of temporal evolution.

Compared the TRMM 3B43 two versions with observational data, the general description of the satellite-based data highlights the existence of similarities and discrepancies with observational data, not only in terms of spatial and time distribution but also in terms of total amount of precipitation. Such disagreement between both satellite-based data and observational data could be due to the difference in their original resolutions. Anyway, this result reasserts the aforementioned comment about the necessity to take into account the comparison of satellite-based data. 


\section{CONCLUSIONS}

A comprehensive evaluation has been performed to examine the performance of satellite-based data and observational data, and monthly precipitation has been constructed on a $0.25^{\circ}$ latitude/longitude grid over Tianshan mountainous regions from 1998 to 2010. The quality of data was evaluated by intercomparisons and cross-validations test. The qulaity of the precipitation estimate degrades as the rain guage being used sparser, and retrieval has almost the same quality based on monthly rain gauge data. It is also important to mention that other factors like wind, evaporation, terrain, among other can also affect the precipitation estimation.

\section{ACKNOWLEDGMENT}

This study was supported by the National Natural Science Foundation of China (41130638, 41130641 and 41001039), the Global Change Research Program of China (973 Programme) (No. 2010CB951401), the Knowledge Innovation Program of the Chinese Academy of Sciences under Grant (No.KZCX2-YW-GJ04).

\section{REFERENCES}

[1] E. E. Elizabeth, E. J. John, k. Chris, "Comparison of near real time precipitation estimates from satellite observations and numerical models." American Meteorological Society, 2007, pp. 47-64.

[2] D. K. Barry, E. F. Gregory, "A comparison of techniques to produce quantile estimates of heavy rainfall in arid and mountainous environments: a test case in western Texas." Journal of Arid Environments, 2000, 44: 267-275.

[3] P. Goovaerts, "Geostatistical approaches for incorporating elevation into the spatial interpolation of rainfall." Journal of Hydrology, 2000, 228, 113-129.

[4] P. P. Xie, A. Yatagai, M. Chen, T. Hayasaka, Y. Fukushima, C. Liu, and S. Yang, 2006: A gauge-based analysis of daily precipitation over East Asia. American Meteorological Society, 2007, 8:607-626.

[5] H. B. Ling, H. L. Xu, J. Y. Fu, Q. Q. Zhang, X. W. Xu, "Analysis of temporal-spatial variation characteristics of extreme air temperature in Xinjiang, China." Quaternary International, 2012, 1-13.

[6] X. M. Li, F. Q. Jiang, L. H. Lia, G. G. Wang, "Spatial and temporal variability of precipitation concentration index, concentration degree and concentration period in Xinjiang, China." International Journal of Climatology, 2011, 31:16791693. 